\title{
Fracturas triplanas de tobillo. A propósito de un caso.
}

DOI: http//dx.doi.org/10.37315/SOTOCAV20212855613

SÁNCHEZ LOSILLA C, BADÍA FERRANDO P, JARA GARCÍA F.

CONSORCIO HOSPITAL GENERAL UNIVERSITARIO VALENCIA.

\section{Resumen.}

Las fracturas triplanas de la tibia distal son un tipo complejo de lesión ósea que ocurre en la adolescencia, antes del cierre de la fisis tibial distal. Corresponden del 6 al $10 \%$ de las fracturas epifisarias del adolescente; y del 5 al $10 \%$ de las lesiones tibiales intrarticulares en pediatría. El objetivo de este trabajo es presentar un caso de fractura triplanar de epífisis distal de tibia tratado mediante tornillos canulados.

Palabras clave: Triplane fracture, distal tibia, ankle fracture

\section{Summary.}

Triplan distal tibial fractures are a complex type of bone injury that occurs in adolescence, prior to closure of the distal tibial physis. They correspond to 6 to $10 \%$ of adolescent epiphyseal fractures; and 5 to $10 \%$ of intra-articular tibial injuries in pediatrics. The objective of this work is to present a case of triplanar fracture of the distal tibial epiphysis treated with cannulated screws.

\section{Correspondencia:}

Correspondencia:

Cristina Sánchez Losilla

csl_cs@hotmail.com 


\section{Introducción}

La fractura triplanar de la epífisis distal de tibia es una fractura compleja. Su patrón que desafía la categorización simple usando el sistema de Salter-Harris. La fractura cumple un criterio de tres planos, con fracturas en el plano sagital, transversal y coronal. Se han descrito numerosas variaciones, incluidas las de dos, tres y de cuatro fragmentos; afectación medial y lateral; y extra- e intraarticulares ${ }^{1-3}$.

La energía del traumatismo es más probable que lesione las estructuras fisarias más débiles que los ligamentos.

La epífisis tibial distal es el segundo sitio más común de fractura epifisaria en la población pediátrica, solo superado por el radio distal. Landing y Danielsson ${ }^{3}$ informaron que sean los más comunes. La fractura epifisaria de tibia distal representa del $5 \%$ al $10 \%$ de las lesiones intraarticulares del tobillo en edad pediátrica, con un incidencia ligeramente mayor en niños que en las niñas.

El objetivo de este trabajo es presentar un caso de fractura triplanar de epífisis distal de tibia tratado mediante tornillos canulados.

\section{Caso Clínico}

\section{- $\quad$ ANAMNESIS:}

Paciente mujer de 12 años de edad, sin antecedentes personales de interés. Acude al Servicio de Urgencias por dolor e impotencia funcional del tobillo derecho tras sufrir un mecanismo de eversión forzada mientras jugaba al fútbol.

\section{EXPLORACIÓN FÍSICA:}

A la exploración física, destaca la presencia de dolor y tumefacción en la región antero-medial del tobillo derecho, con movilidad limitada y hematoma en cara medial. No se objetivan alteraciones vásculo-nerviosas distales.

\section{PRUEBAS COMLEMENTARIAS:}

Se realiza un estudio radiográfico completo (proyección anteroposterior, lateral y mortaja), así como una tomografía computarizada del tobillo derecho.

\section{DIAGNÓSTICO:}

Se diagnostica de fractura triplana en dos fragmentos a nivel de tibia distal, sin afectación del peroné. Esta fractura se corresponde con un tipo III de Salter y Harris en la proyección anteroposterior y un tipo II de Salter y Harris en la proyección lateral. (Figs. 1A y 1B).
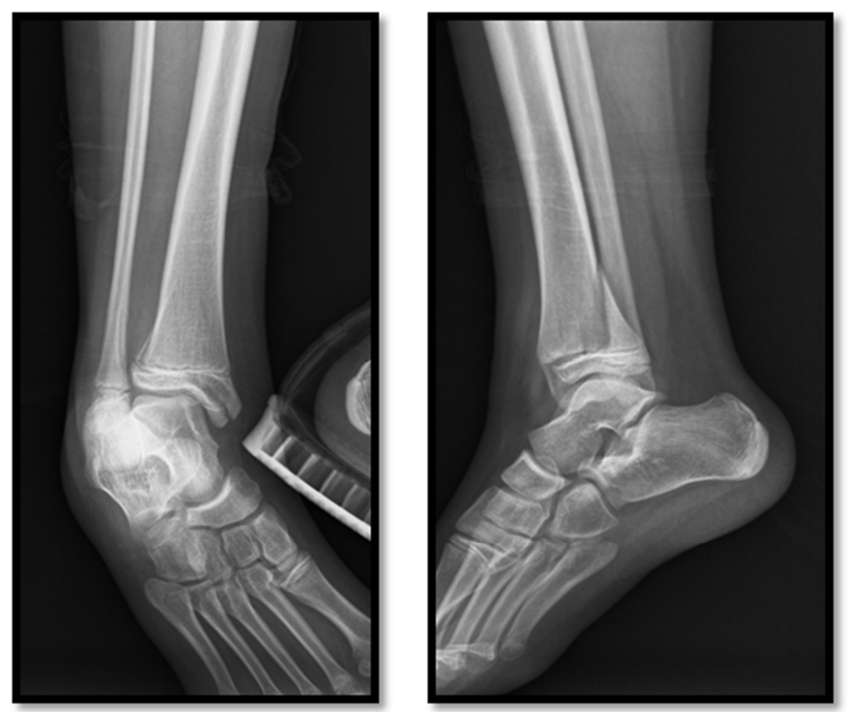

Figura 1A y 1B: proyección anteroposterior y lateral de fractura triplana en dos fragmentos.

\section{TRATAMIENTO:}

Se decide realizar tratamiento quirúrgico mediante reducción y estabilización de la fractura con dos tornillos canulados de esponjosa de rosca parcial de $4 \mathrm{~mm}$ de forma percutánea; ambos colocados de manera anteroposterior proximales a la fisis. Se comprueba la correcta reducción de la fractura y la correcta colocación del material de osteosíntesis mediante escopia intraoperatoria. Finalmente se inmovilizó la pierna con una férula posterior, manteniendo la articulación del tobillo a $90^{\circ}$. (Figs $2 \mathrm{~A}$ y $2 \mathrm{~B}$ )

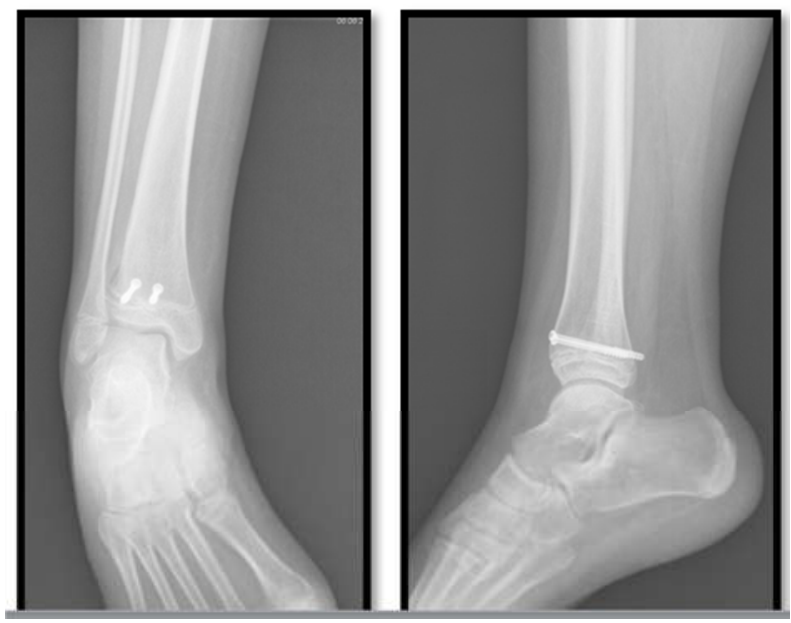

Figura 2A y 2B: fractura triplana tras tratamiento quirúrgico mediante 2 tornillos canulados.

\section{EVOLUCIÓN:}

Después de seis semanas de inmovilización, el paciente comenzó la rehabilitación activa y pasiva del tobillo derecho, así como la carga total de manera progresiva. 
La evolución clínica fue satisfactoria con un rango de movilidad completo, ausencia de dolor y una correcta reducción anatómica en el último control radiográfico.

\section{Discusión}

Las fracturas triplanas de la tibia distal son un tipo complejo de lesión ósea que ocurre en la adolescencia, antes del cierre de la fisis tibial distal. Corresponden del 6 al $10 \%$ de las fracturas epifisarias del adolescente; y del 5 al $10 \%$ de las lesiones tibiales intrarticulares en pediatría ${ }^{1,4}$.

La principal preocupación en el momento de tratar las fracturas triplanas es conservar la integridad de la superficie articular del tobillo, para evitar cambios degenerativos articulares. Por tanto, en las fracturas extrarticulares y no desplazadas, optaremos por un manejo conservador mediante reducción cerrada e inmovilización con férula posterior; que conseguiremos mediante tracción con posterior rotación interna y supinación. Tras la reducción cerrada, siempre realizaremos control con TC, para comprobar que efectivamente hemos conseguido una reducción anatómica.

Por el contrario, optaremos por un tratamiento quirúrgico mediante AK o tornillos, cuando tengamos un escalón o desplazamiento mayor de $2 \mathrm{~mm}$ en la superficie articular ${ }^{5,6}$.

Se recomienda el seguimiento de estos pacientes hasta que se completa la madurez ósea.

\section{Bibliografía}

1. El-Karef E, Sadek HI, Nairn DS, Aldam CH, Allen PW. Triplane fracture of the distal tibia. Injury 2000; 31:729-36.

2. Schnetzler KA, Hoernschemeyer D. The pediatric triplane ankle fracture. J Am Acad Orthop Surg 2007; 15:738-47.

3. Landin LA, Danielsson LG. Children's ankle fractures: Classification and epidemiology. Acta Orthop Scand 1983; 54:63440.

4. Peterson CA, Peterson HA. Analysis of the Incidence of Injuries to the Epiphyseal Growth Plate. J Trauma 1972; 12:27581.

5. Hirsch M, Flores F. Comprendiendo las fracturas triplanares de tibia distal. Revista Chilena de Radiografía 2016 ; 22 (3):114-20.

6. Blount WP. Fractures in Children. Baltimore: Williams and Wilkins, 1955. 